\title{
Infant Sedation, AE
}

National Cancer Institute

\section{Source}

National Cancer Institute. Infant Sedation, AE. NCI Thesaurus. Code C154926.

An adverse event in a newborn characterized by a state of a lowered level of consciousness. 